\title{
La Competitivite Du Port De Dakar A Partir De L'etude De La Desserte De Bamako : Diagnostic Et Amelioration
}

\begin{abstract}
Issakha Dia,
Enseignant-chercheur, Assistant, Laboratoire de Matériaux et d'Energetique (LME), Institut Universitaire de Technologie, Université de Thiès, Sénégal

Salif Gaye,

Enseignant-chercheur, Professeur titulaire, Laboratoire de Matériaux et d'Energetique (LME), Institut Universitaire de Technologie, Université de

Thiès, Sénégal
\end{abstract}

Doi:10.19044/esj.2019.v15n26p193 URL:http://dx.doi.org/10.19044/esj.2019.v15n26p193

\section{Résumé}

La croissance du commerce international sollicite grandement les ports les poussant à déployer des stratégies pour être compétitifs. Les ports de l'Afrique de l'ouest n'en font pas exception. La compétitivité d'un port peut être appréhendée au niveau des tarifs, de la qualité de service, du temps de passage, etc., certes, mais elle dépend beaucoup aussi de sa desserte terrestre. La présente étude, cherche à montrer la place du port de Dakar dans le dispositif logistique du Mali en le comparant au port d'Abidjan avant de présenter la desserte de Bamako depuis le port de Dakar. La revue documentaire, la collecte des données, leur traitement et l'analyse des résultats constituent les principales articulations de la démarche méthodologique adoptée. Pour ce qui est de la connaissance des parties prenantes de la desserte vers Bamako, des descentes sur le terrain ont été effectuées régulièrement en 2018 et 2019. Elles ont été ponctuées de mesures (sur le flux du transport routier, sur le temps de contrôle des agents de route, etc.) d'enquêtes au près d'agents du port, de Dakar-Bamako-Ferroviaire (DBF), d'AGEROUTE et d'autres acteurs le long du corridor. Les résultats obtenus montrent d'abord au niveau de la comparaison entre les ports de Dakar et d'Abidjan par rapport au client malien, que Dakar, bien que ne disposant pas des meilleures infrastructures, propose les meilleurs tarifs aux chargeurs. Par rapport à la desserte routière sur le corridor, du côté routier, de gros efforts sont faits au niveau infrastructurel, mais le nœud du problème demeure la gouvernance où on constate encore une perte de temps et des perceptions illicites. Le transport ferroviaire est important dans le dispositif de desserte du port de Dakar mais 
il souffre d'une dégradation très avancée et des actes concrets pour le remettre en marche tardent.

Mots clés: Bamako, compétitivité, desserte routière, desserte ferroviaire, Port de Dakar

\title{
The Competitiveness of the Port of Dakar from the Study of the Bamako Dessert: Diagnosis and Improvement
}

\section{Issakha Dia,}

Enseignant-chercheur, Assistant, Laboratoire de Matériaux et d'Energetique (LME), Institut Universitaire de Technologie, Université de Thiès, Sénégal

Salif Gaye,

Enseignant-chercheur, Professeur titulaire, Laboratoire de Matériaux et d'Energetique (LME), Institut Universitaire de Technologie, Université de Thiès, Sénégal

\begin{abstract}
The growth of international trade is being solicited from ports to deploy strategies to be competitive. Africa's ports of Africa is no exception. The competitiveness of a port can be apprehended at the level of the tariffs, the quality of service, the time of passage, etc., certainly, but it also depends a lot on its terrestrial service. The present study, is to show the place of the port of Dakar in the logistics of Mali and the comparison to the port of Abidjan before presenting the service of Bamako from the port of Dakar. The documentary review, the collection of data, their treatment and the analysis of the results constituting the main articulations of the methodological approach adopted. As for the knowledge of the stakeholders of the service to Bamako, field trips were regularly made in 2018 and 2019. They were punctuated by measures (on the road transport flow, on the control time road, etc.), near the agent agent of the port, Dakar-Bamako-Ferroviaire (DBF), AGEROUTE and other actors along the corridor. The results expected at the first level of comparison between the ports of Dakar and Abidjan compared to the Malian customer, that Dakar, as well as the best infrastructures, offer the best tariffs to shippers. We have moved on to infrastructure, but we have not yet solved the problem of governance, but we have found that there is still a waste of time
\end{abstract}


and illicit perceptions. The rail transport is important in the service of the port of Dakar but it is very advanced and the concrete acts are postponed.

Keywords: Bamako, competitiveness, Port of Dakar, road access, rail service

\section{Introduction}

La croissance du commerce international est largement tributaire de l'efficacité du transport maritime. Il pèse plus de $80 \%$ des volumes des marchandises déplacés, soit 10,7 milliards de tonnes/année. L'Afrique n'y contribue qu'à hauteur de $7 \%$ dans les exportations et $5 \%$ dans les importations (UNCTAD, 2018). Pour accroître sa part dans le trafic mondial, l'Afrique investit de gros montants dans son secteur portuaire; ce qui lui a valu une croissance annuelle de 7\% sur la décennie 2007-2017 (Matz, 2017). Les échanges commerciaux entre l'Afrique et ses partenaires portent essentiellement sur ses matières premières, ses sources d'énergie et ses productions agricoles (Champonnière, 2008; Steck, 2004). Selon la CNUCED, elles pèsent en valeur plus de $60 \%$ des exportations dans tous les pays du continent africain, sauf six : l'Afrique du Sud, le Swaziland, le Lesotho, le Maroc et la Tunisie. (CNUCED, 2017). Ces échanges sont interpellatifs sur les objectifs de développement des pays africains qui seront très difficiles à atteindre quand on sait combien sont volatiles les prix des matières premières (Solagral, 2003). Malgré tout, ils sont importants pour l'Afrique, nonobstant la difficulté de leur mise en œuvre. Avec le concours de l'Organisation mondiale du commerce (OMC), les mesures traditionnelles de protection du commerce que constituent les tarifs douaniers et les quotas se relâchent de plus en plus. Elles sont toutefois remplacées par des règlementations techniques nationales qui permettent aux pays d'interdire l'entrée dans leur territoire de produits ne satisfaisant pas à certaines normes. Mais, quoiqu'il en soit, l'Afrique témoigne d'une ferme volonté de faire partie du commerce mondial (avec 46 Etats membres à l'OMC soit $85 \%$ des pays africains et $28 \%$ des pays signataires - OMC, $2019-$-) car elle veut faire partie des jeux de transactions commerciales avec les autres pays du monde. Elle compte ainsi sur ses 80 ports (CEA, 2004) pour interagir avec les autres parties du monde.

L'Afrique de l'ouest, de Dakar à Lagos, compte 15 ports, et pourtant $20 \%$ de la population que couvre cette région sont issues des pays enclavés (Burkina Faso, Mali et Niger). Leur contribution au produit intérieur brut (PIB) mondial qui est de l'ordre de 0,05\% (UNCTAD, 2017) les classent parmi les pays les plus pauvres du monde. Malgré leur faible tribut au commerce international, ces Etats, dont le Mali est un exemple illustratif, ont une absolue nécessité d'être connectés aux réseaux mondiaux de la transaction des flux (Steck, 2004). Pour ce faire, il passe par les portes océanes de ses 
voisins, principalement la Côte d'Ivoire et le Sénégal.

Le Mali, pour ses activités commerciales avec ses collaborateurs européens et asiatiques, nécessite des passages portuaires. C'est ainsi que le port de Dakar a conclu des partenariats public-privé (PPP) lui permettant d'étendre son domaine et d'améliorer ses services afin de mieux répondre aux besoins de ses clients et être ainsi plus compétitif afin de résister aux assauts répétés du port d'Abidjan sur le marché malien. Selon les statistiques des autorités portuaires de 2017, on note une prééminence du port de Dakar avec 2675959 tonnes suivi de celui d'Abidjan avec 821149 tonnes. Le Sénégal est donc la principale porte d'accès maritime du Mali qui plus est le destinataire de 96,4\% des marchandises en transit du port de Dakar. Ces résultats sont certes très importants, mais l'on ne sécurisera cette avance que par une amélioration de la compétitivité du port de Dakar. C'est dans ce cadre que des accords de partenariats public-privé (PPP) ont été conclus par le port autonome de Dakar. L'objectif est d'étendre son domaine et d'améliorer ses services afin d'être plus compétitif et continuer à rester le leader sur le marché malien. Mais est-ce seulement les infrastructures, les équipements et les services qui garantissent la compétitivité d'un port ? Notre analyse montrera comment par une bonne gestion des corridors d'un port (gouvernance, fluidité), on peut contribuer à sa compétitivité.

\section{Matériels et méthodes \\ 1.1. Cadre géographique}

Compte tenu du tonnage croissant de marchandises maliennes traitées au port de Dakar depuis le début de la crise ivoirienne en 2002, le choix du Mali est clair. Bamako, grace aux deux corridors nord et sud (DakarTambacounda-Kidira-Bamako et Dakar-Tambacounda-Kédougou-Bamako ) qui le desservent, est respectivement accessible à 1382 et $1225 \mathrm{~km}$ (figure1), mais aussi par le chemin de fer, qui est un patrimoine commun entre le Sénégal et le Mali (figure2). 


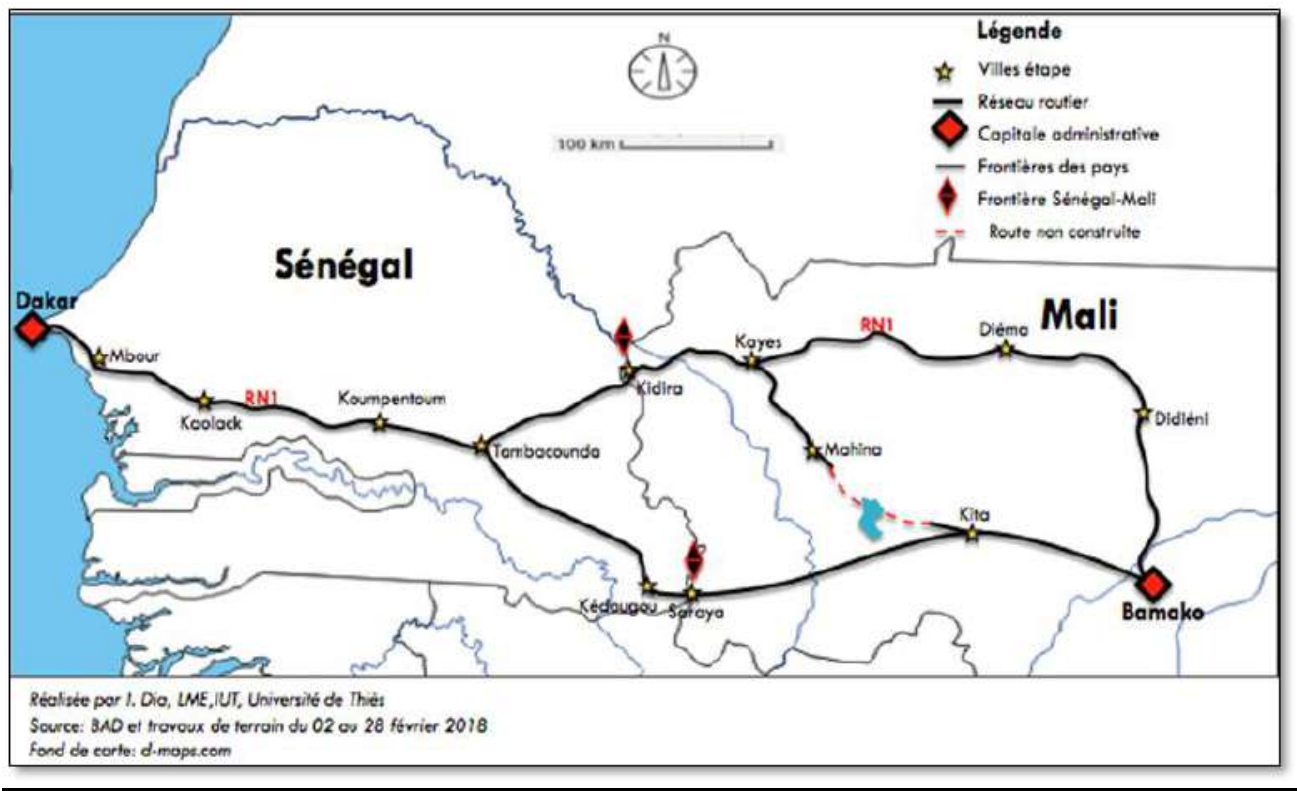

Figure 1 : Corridor routier Dakar-Bamako (axe Nord, axe Sud et projet d'axe centre)

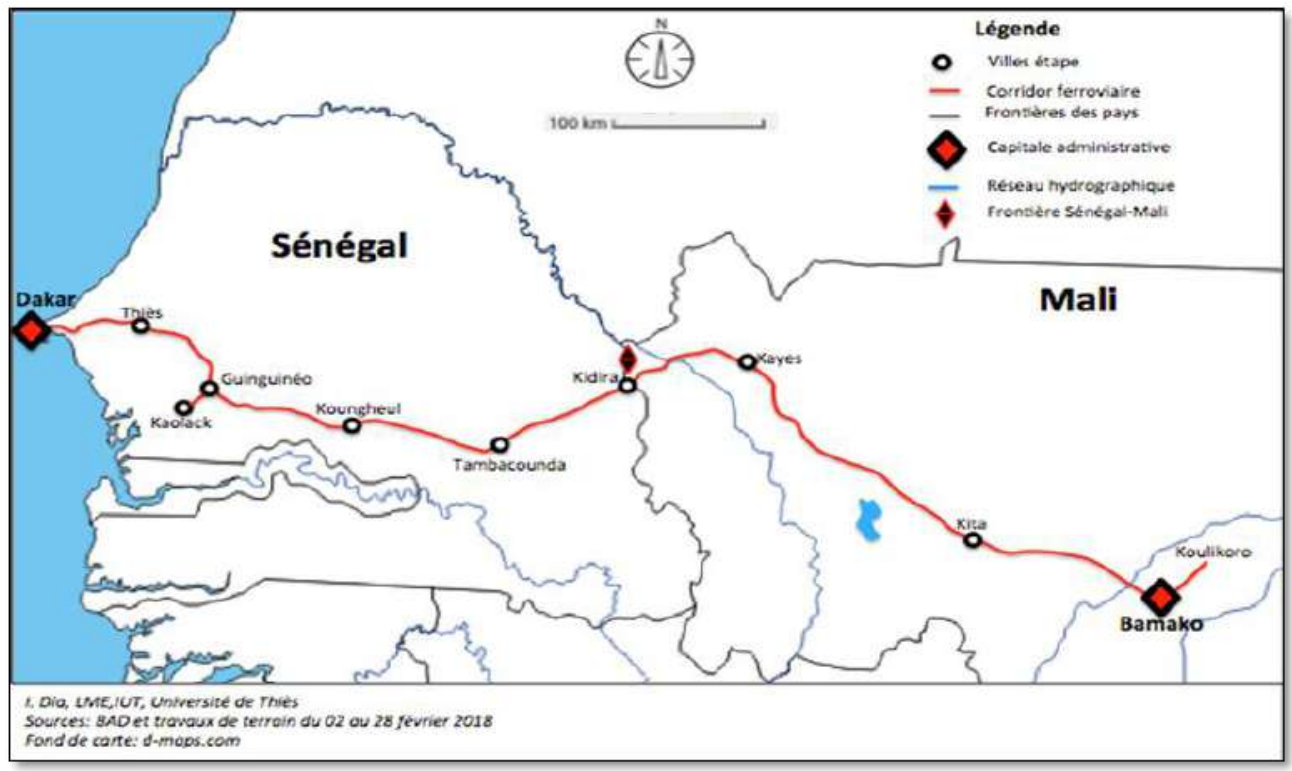

Figure 2 : Corridor ferroviaire Dakar-Bamako

\subsection{Techniques de collecte de données}

Cette étude s'est appuyée sur trois méthodes classiques de collecte de données.

D'abord une revue documentaire qui montre l'intérêt que suscite les études sur la compétitivité portuaire de par le monde. La plupart des résultats mettent en avant une concurrence interportuaire où les parties prenantes 
choisissent un port, selon des critères définis (Aronietis et al., 2010 ; Merk et Comtois, 2012 ; Onwuegbuchunam, 2013 ; Lihoussou 2014). Dans d'autres analyses, la compétitivité du port est mesurée par rapport à la gestion de son hinterland. Les résultats des travaux de Maloni et Jackson (2005), Harding et al. (2007), Zhang (2008), OECD/ITF (2008) démontrent que la croissance des ports et de ses parts de marché pâtissent lorsque les niveaux de congestion au niveau de l'arrière-pays sont élevés. Ceci doit rappeler que les mutations dans les ports sont importantes, certes, mais la gestion des flux sur l'hinterland l'est tout autant (Harding et al., 2007 ; Yao Beli et Konan Koffi, 2018 ; Dicko, 2018).

Cette revue documentaire est complétée par la visite des sites internet des ports de Dakar et d'Abidjan afin de recueillir des données sur leurs trafics et leurs caractéristiques physiques.

La collecte d'informations s'est poursuivie dans les structures qui s'intéressent aux questions de transport de marchandises telles que le port de Dakar, le Conseil sénégalais des chargeurs (COSEC), le Conseil malien des chargeurs et dans les locaux de Dakar-Bamako-Ferroviaire (DBF) de Dakar et de Thiès ainsi que ceux qui sont en charge de la gestion routière : Agence de gestion des routes (AGEROUTE), les postes de pesage, sans oublier la douane qui occupe une place essentielle dans le transport international.

Dans le but de confronter les informations issues de la revue documentaire à la réalité, des enquêtes de terrain ont été organisées. Elles ont été ponctuées de courts et longs séjours allant d'un à 45 jours entre Dakar, Guinguinéo, Kaolack, ou un peu plus loin, à Tambacounda, Kidira, Kédougou et hors des frontières sénégalaise à Kayes et Bamako, durant les années 2018 et 2019. Ainsi, des questionnaires et guides d'entretien ont été administrés. Dans les 3 entreprises de transport et de prestations portuaires visitées, les processus des passages portuaires (navire et marchandises) ont été expliqués et par la même occasion, avec les chefs de flotte, la question du transport routier a été largement débattue. Ces entreprises sont le TAD (Transports Amadou Dièye), la SOCOTRA (Société de consignation et de transit) et la SDV Mali. Dans un souci d'élargir le champ d'analyse, 02 transporteurs informels sénégalais ont été également interviewés. Les entretiens se sont continués avec le transport ferroviaire à DBF (Thiès et Kayes - respectivement Sénégal et Mali) où 02 responsables logistiques et 10 chefs de gare ont été interrogés. Ensuite au COSEC et à l'AGEROUTE et à Afrique pesage, où respectivement 02,02 et 10 agents ont été questionnés. Les agents de police (05), de douane (05) et de gendarmerie (05) le long des corridors et au niveau des postes frontières ont également répondu à nos questions. Les transporteurs, par la voie de leur syndicat «Union des routiers du Sénégal », ont aussi réagi aux questions posées. Enfin, 50 camionneurs ont été interrogés afin de partager leurs expériences du circuit car ils sont présents dans tous les théâtres 
d'opération : port, postes de pesage, aires de repos improvisées ou sur le bas côté de la route à cause d'une panne ou d'un simple stationnement anarchique.

Enfin, pour faire ressortir la compétitivité du port de Dakar, son passage portuaire a été comparé avec celui d'Abidjan dans le but de confronter les résultats liés aux délais et aux coûts de passage portuaire qui sont évalués à partir du volume (navire) à l'exeption de quelques tarifs qui sont forfaitaires : $\mathrm{V}=\mathrm{L}$ x 1 x Tme ;

- $\quad$ L : longueur hors tout du navire en mètre ;

- 1 : largeur maximale du navire en mètre;

- $\quad$ Tme : tirant d'eau maximale du navire en mètre.

\section{Résultats et interprétations}

\subsection{Le séjour portuaire}

Le port autonome de Dakar est un port en eau profonde capable d'accueillir des navires à fort tirant d'eau et fonctionne 24 heures/24 et 7jours/7. Situé à la croisée des routes maritimes reliant 1'Amérique du Sud à l'Europe, l'Amérique du Nord à l'Afrique du Sud, le port de Dakar, bénéficie d'une situation géographique exceptionnelle. En plus, sa position de port le plus avancé de l'Afrique continentale est un atout certain lui permettant de pouvoir accueillir des navires venant de l'Amérique du nord et de l'Europe 2 à 3 jours par rapport à ses concurrents situés dans le golfe de Guinée (port autonome de Dakar, 2018) à l'exemple du port d'Abidjan. Malgré une position moins favorisée par la nature (variations des marées qui rendent les accostages délicats), le canal deVridi qui relie le port à la mer a été complètement rénové par China Harbour Engeneering company (CHEC) suite à un contrat de concession, ce qui permet au port d'Abidjan de recevoir des navires de plus grandes dimensions qu'il y a quelques années (port autonome d'Abidjan, 2018).

Ces deux ports misent beaucoup sur le marché malien. Pour le port de Dakar, Bamako est son hinterland naturel depuis le début de la crise ivoirienne en 2002. Le Mali est le seul pays parmi ceux qui sont frontaliers au Sénégal à ne pas disposer d'une façade maritime et est la destination quasi-exclusive du trafic de transit du port de Dakar. Pour la Côte d'ivoire, c'est surtout une question de reconquête de son marché après la crise qu'a connu le pays entre 2002 et 2012. Avant la crise, le port d'Abidjan assurait entre 60 et $75 \%$ des échanges extérieurs du Mali selon les années (Steck, 2004). Parmi les stratégies mises en place pour reconquérir ce marché, des mesures incitatives susceptibles de redonner confiance aux chargeurs (Flavient, 2018). 
Tableau 1 : Fiche technique des ports de Dakar et d'Abidjan pour la desserte de Bamako

\begin{tabular}{|c|c|c|}
\hline Désignations & Port de Dakar & Port d'Abidjan \\
\hline Linéaire de quai $(\mathrm{km})$ & 10 & 06 \\
\hline Poste conteneurs & 03 & 05 \\
\hline Longueur du chenal dragué (m) & 200 & 2700 \\
\hline $\begin{array}{l}\text { Terre plein total (TAC inclus) } \\
\text { en ha }\end{array}$ & 117 & 170 \\
\hline $\begin{array}{l}\text { TAC (terminal à conteneurs) en } \\
\text { ha }\end{array}$ & 17 & 34 \\
\hline Entrepôts maliens $\left(\mathrm{m}^{2}\right)$ & 51000 & 34000 \\
\hline $\begin{array}{lll}\text { Hangars } & \text { destinés } & \text { aux } \\
\text { marchandises } & \text { maliennes }\end{array}$ & 9300 & 13000 \\
\hline $\begin{array}{l}\text { Trafic global (en tonnes) en } \\
2018\end{array}$ & 19220341 & 24177261 \\
\hline Trafic total EVP en 2018 & 697336 & 674624 \\
\hline $\begin{array}{l}\text { Trafic en transit (en tonnes) } \\
\text { pour le Mali en } 2018\end{array}$ & $\begin{array}{l}2635709 \text { soit } 13,7 \% \text { du } \\
\text { trafic global }\end{array}$ & $\begin{array}{l}537028 \text { soit } 2,2 \% \text { du } \\
\text { trafic global }\end{array}$ \\
\hline $\begin{array}{l}\text { Poste à quai prioritairement } \\
\text { réservé au trafic malien }\end{array}$ & $\mathbf{0 2}$ & - \\
\hline Connexion voie ferroviaire & $\begin{array}{l}\text { Embranchement } \\
\text { ferroviaire Mali }\end{array}$ & - \\
\hline
\end{tabular}

Source : port autonome de Dakar, port autonome d'Abidjan, observatoire des transports 2014 et auteurs

A l'analyse du tableau 1, il apparaît clairement qu'au delà du linéaire de quai, des entrepôts réservés au maliens pour leur trafics, des trafics en transit en destination du Mali et du trafic en EVP, des 02 postes à quai du môle 3 et de l'embranchement ferroviaire en destination du Mali, le port d'Abidjan dépasse le port de Dakar sur les 12 critères analysés. Cette situation instable du port de Dakar est accentuée par les grands travaux (certains sont déjà livrés comme le canal de Vridi, et d'autres en cours comme la modernisation des quais et la construction d'un second terminal à conteneurs) et qui devraient être livrés en 2020 selon le directeur du port Hien Sié. Cette situation de monopole peut, en un temps record, changer en faveur de la Côte d'ivoire d'autant plus qu'elle travaille sur un autre pion à savoir le port de San Pedro. Les autorités du port de San Pedro ont signé un protocole avec la Compagnie malienne pour le développement des textiles (CMDT). Dans ce cadre, le port a engagé des travaux pour la mise en place d'entrepôts maliens en Côte d'ivoire (EMACI) et de bureaux destinés au conseil malien des chargeurs. En outre, un comité technique a été mis en place en vue de mettre en œuvre un projet d'aménagement routier pour faciliter le transport sur le corridor Bamako- Zantiebougou-Boundiali-San Pedro (Flavient, 2018)

Par ailleurs, il faut reconnaître que le port de Dakar a bien compris les 
attentes du client malien, car depuis la fin de la crise ivoirienne, plus de six ans sont passés, et il maintient encore sa position avec de larges scores sur les trafics en transit (tableau 1). Parmi les éléments technico-commerciaux qu'utilisent le port de Dakar pour fidéliser le client malien, on peut noter les entrepôts Maliens au Sénégal (EMASE), un embranchement ferroviaire au plus près des espaces de chargement, un abattement de $10 \%$ pour la location de hangars et $50 \%$ sur les redevances maritimes, des délais de franchise de l'ordre de 21 jours pour les marchandises diverses et de 12 jours pour les voitures etc. Tout cela explique les rapports commerciaux étroits entre les deux pays et la place de choix qu'occupe le port de Dakar dans le système logistique du commerce extérieur malien.

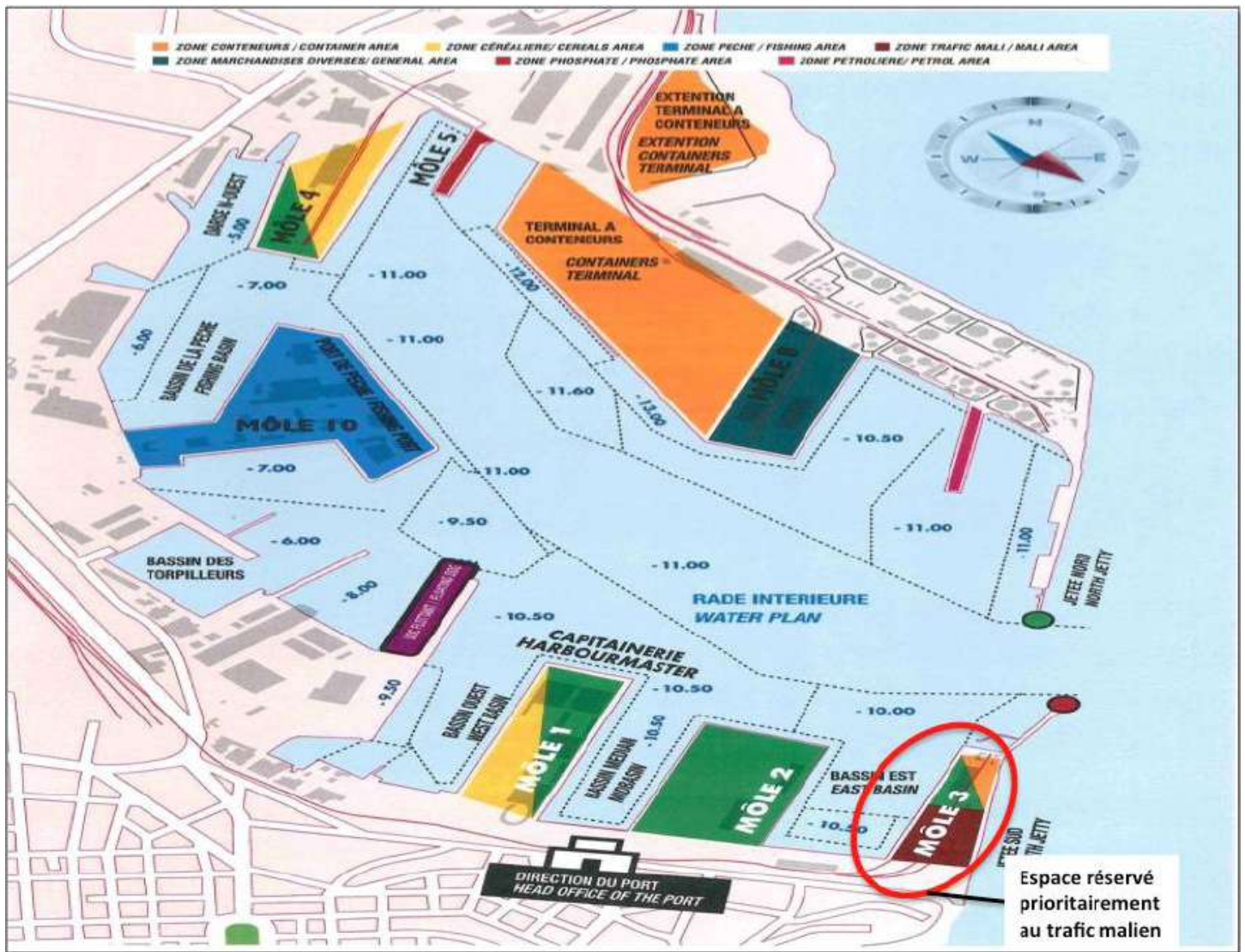

Figure 3 : Plan de masse du port de Dakar

Source : Adapté de port autonome de Dakar

\subsubsection{Le coût de passage portuaire}

Les ports sont choisis par les clients en fonction de leurs infrastructures, de la qualité de service (vitesse de rotation des navires, temps de passage des marchandises etc.) proposée et des tarifs appliqués. 
Les services facturés lors du passage d'un navire commercial dans les ports de Dakar et d'Abidjan sont consignés dans le tableau 2. Tous les tarifs sont calculés sur la base du volume à part la taxe au navire et le droit d'entrée au port qui sont fixes et forfaitaires, mais aussi la taxe International Ship and Port Facility Security (ISPS) au port d'Abidjan qui est évalué à 10\% par rapport au compte d'escale.

Tableau 2 : Coûts des services portuaires pour les navires commerciaux dans les ports de

Dakar et d'Abidjan.

\begin{tabular}{|c|c|c|}
\hline Désignation & $\begin{array}{l}\text { Tarifs au port de Dakar } \\
\text { en FCFA }\end{array}$ & Tarifs au port d'Abidjan en FCFA \\
\hline Pilotage (pilotage) & $11,8 / \mathrm{m}^{3}$ & $\begin{array}{l}\text { avec un minimum de perception de } \mathbf{3 2 7} \\
\mathbf{9 7 8} \text { pour les catégories de } 1 \text { à } 6 \text { et } \mathbf{1 9 6} \\
\mathbf{7 8 7} \text { pour la catégorie } 7 \text {; }\end{array}$ \\
\hline $\begin{array}{l}\text { Amarrage } \\
\text { (mooring/unmooring) }\end{array}$ & $1,6 / \mathrm{m}^{3} /$ opération & $\begin{array}{l}\text { Tarification par classe de navire }(7 \\
\text { catégories) selon les tranches de volumes } \\
\text { avec un minimum de perception de } \mathbf{2 2 9} \\
\mathbf{5 8 4} \text { pour les catégories de } 1 \text { à } 6 \text { et } \mathbf{1 1 4} \\
\mathbf{7 9 2} \text { pour la catégorie } 7 \text {; }\end{array}$ \\
\hline Droits de port (port dues) & $14,16 / \mathrm{m}^{3} /$ jour & $\begin{array}{l}\text { Tarification par classe de navire }(7 \\
\text { catégories) selon les tranches de volumes } \\
\text { avec un minimum de perception de } \mathbf{1 8 0} \\
\mathbf{3 3 8} \text { pour les catégories de } 1 \text { à } 6 \text { et } \mathbf{6 5 5 0 0} \\
\text { pour la catégorie } 7 \text {; }\end{array}$ \\
\hline $\begin{array}{l}\text { Taxe au navire }(\operatorname{tax} \text { on } \\
\text { vessel) }\end{array}$ & $\begin{array}{l}\mathbf{9 8 3 9 3 5 .} \\
\text { C'est un montant } \\
\text { forfaitaire appliqué à tous } \\
\text { les navires par l'agence } \\
\text { nationale des affaires } \\
\text { maritimes (ANAM) }\end{array}$ & - \\
\hline Remorquage (towage) & $\begin{array}{l}\text { défini par intervalle de } \\
\text { volume selon la durée et } \\
\text { la période d'intervention } \\
\text { (voir document de l'union } \\
\text { des remorqueurs de Dakar } \\
\text { - URD -.) }\end{array}$ & - \\
\hline $\begin{array}{l}\text { Taxe de sécurité ISPS } \\
\text { (International Ship and Port } \\
\text { Facility Security) }\end{array}$ & $\begin{array}{l}\text { défini par intervalle de } \\
\text { volume en } \mathrm{m}^{3}\end{array}$ & $\begin{array}{l}10 \% \text { du montant total du compte } \\
\text { d'escale; }\end{array}$ \\
\hline Taxe d'environnement & - & $\begin{array}{l}13000 \text { pour tout type de navire à } \\
\text { l'exclusion des pétroliers qui sont tarifés } \\
\text { par intervalle de volume; }\end{array}$ \\
\hline $\begin{array}{l}\text { Droit d'entrée au port } \\
\text { (custom entry manifest) }\end{array}$ & $\begin{array}{l}41300 \text { (c'est un montant } \\
\text { forfaitaire appliqué à tous } \\
\text { les navires) }\end{array}$ & $\begin{array}{l}\text { Tarification par classe de navire }(7 \\
\text { catégories) selon les tranches de volumes } \\
\text { avec un minimum de perception de } \mathbf{3 8 0} \\
\mathbf{4 5 5} \text { pour les catégories de } 1 \text { à } 6 \text { et } \mathbf{2 9 5} \\
\mathbf{1 8 0} \text { pour la catégorie } 7 .\end{array}$ \\
\hline
\end{tabular}

Source : I. Dia, d'après les barèmes de redevances des ports de Dakar et d'Abidjan, note sur la redevance portuaire ISPS, URD, ANAM. 
C'est en référence à ces tarifs (tableau 2) que le consignataire, qui est le représentant à terre du navire, établit une facture (frais de ports et facture consignation) à l'attention de l'armateur. Les tarifs, lors d'une opération de transport internationale multimodale, font partie du triptyque (Coût-QualitéDélai) d'éléments prioritaires qui influencent le choix de l'armateur pour un port donné (Fassio et Le Mestre, 2009). Les ports de Dakar et d'Abidjan suivent le même protocole pour la facturation des navires à quelques exceptions près. A Dakar, contrairement à Abidjan, on facture les taxes au navire et le remorquage. Mais la grande différence entre ces deux ports est la base taxable. Si à Dakar, on facture directement à l'unité de volume, à Abidjan c'est une catégorisation des navires qui est faite par rapport à leurs tailles et conséquemment à cela, les tarifs sont fixés.

Le débarquement des marchandises ne peut se faire qu'à la suite des services portuaires sur le navire. Elles suivent, également, une autre grille de facturation établie en plusieurs rubriques. Les hypothèses d'analyse choisies sont des chargements de $30 \mathrm{t}$ pour quelques marchandises parmi les plus importées au Mali et également du riz en conteneur. Ces chargements sont en transit au port de Dakar (tableau 3).

Tableau 3 : Coûts des prestations rendues sur quelques marchandises en transit au port de Dakar (riz, engrais, fer à béton) en FCFA.

\begin{tabular}{|c|c|c|c|c|}
\hline & Riz (vrac) & Engrais & Riz (conteneur) & Fer à béton \\
\hline Consignation & $8,5 \%$ & $8,3 \%$ & $7,1 \%$ & $4,8 \%$ \\
\hline Avis d'arrivée & 10000 & 10000 & 10000 & 10000 \\
\hline $\begin{array}{l}\text { Imprimé } \\
\text { (document) }\end{array}$ & 1500 & 1500 & 1500 & 1500 \\
\hline Timbre BL & 8000 & 8000 & 8000 & 8000 \\
\hline Communication & 10000 & 10000 & 10000 & 10000 \\
\hline Manutention & $40,8 \%$ & $42,3 \%$ & $47,6 \%$ & $54,9 \%$ \\
\hline Acconage & 102600 & 109350 & 125025 & 337590 \\
\hline Relevage & & & 18250 & \\
\hline $\begin{array}{l}\text { Redevances } \\
\text { portuaires }\end{array}$ & $1 \%$ & $1 \%$ & $3,7 \%$ & $19,9 \%$ \\
\hline $\begin{array}{l}\text { Redevance de } \\
\text { passage }\end{array}$ & 2595 & 2700 & 11000 & 122160 \\
\hline Transit & $49,7 \%$ & $48,4 \%$ & $41,6 \%$ & $20,4 \%$ \\
\hline $\begin{array}{l}\text { Forfait } \\
\text { Honoraires } \\
\text { agréées en } \\
\text { douanes (HAD) }\end{array}$ & 130000 & 130000 & 130000 & 130000 \\
\hline Total & 256695 & 263550 & 305805 & 619250 \\
\hline
\end{tabular}

Source: I. Dia, d'après Barème de redevances pour le port de Dakar, enquêtes réalisées en février 2018 au port et dans des entreprises de consignation, 
A la lecture du tableau 3, on constate des coûts très faibles pour les redevances portuaires. Sur certains produits, elles ne représentent que $1 \%$ de la facture globale de passage portuaire. Les tarifs de la manutention et du transit occupent quasiment, pour tous les produits, plus de $80 \%$ de la facture. Ils représentent les quatre cinquième de la facture, mais sont souvent sujets à beaucoup de retours négatifs de la part des opérateurs qui leur reprochent un défaut de transparence et un problème de lisibilité dans les barèmes appliqués. Pour rappel, le transitaire, dans une opération de passage portuaire, est une personne physique ou morale mandatée par le chargeur pour accomplir des formalités de dédouanement d'une marchandise. Comme comparé dans les grilles tarifaires des entreprises étudiées on a constaté une certaine flexibilité des tarifs appliqués pour un même service ; raison pour laquelle dans cette analyse on a appliqué un montant forfaitaire (basé sur les tarifs appliqués dans ces entreprises). La manutention quant à elle se matérialise par les opérations de chargement et de déchargement des navires. Ce que les clients ne comprennent pas souvent sur leurs factures, c'est que le conditionnement et la nature du produit influent fortement sur la tarification. Par exemple, le riz en conteneur et le fer à béton ont une manutention plus chère que le riz en vrac.

$\mathrm{Si}$ on compare les tarifs de redevances portuaires avec les mêmes marchandises (poids et catégories identiques) transitant au port d'Abidjan, on constate que les tarifs sont beaucoup plus abordables à Dakar (tableau 4) allant de 2,5 fois moins cher pour le riz en vrac à 9,3 fois moins cher pour l'engrais.

Tableau 4 : Tarifs des redevances des marchandises transitant aux ports de Dakar et d'Abidjan en FCFA.

\begin{tabular}{|c|c|c|c|c|c|}
\hline & $\begin{array}{l}\text { Riz } \\
\text { /Tonne }\end{array}$ & (vrac) & Engrais/Tonne & Riz (conteneur) & $\begin{array}{l}\text { Fer à } \\
\text { béton/Tonne }\end{array}$ \\
\hline Dakar & 86,5 & & 90 & 11000 & 863 \\
\hline Abidjan & 220,5 & & 840 & 10000 & 1190 \\
\hline
\end{tabular}

Source: I. Dia, d'après Barème de redevances pour les port de Dakar et d'Abidjan.

Ces deux tarifications croisées dénotent de la volonté de l'autorité du port de Dakar d'aménager des tarifs favorisant la compétitivité sa structure.

\subsubsection{Les délais de passage portuaire}

Les délais de passage portuaire sont en général difficiles à déterminer. Ils sont la conséquence de l'addition des temps passés au niveau de chaque étape. Les informations que l'on y collecte viennent souvent des conseils des chargeurs, les consignataires, surtout quand les résultats ne vont pas en leur faveur préfêrent ne pas les divulguer. Le port de Dakar connaît des délais de passage portuaires longs. Une analyse chronologique des temps de transit de 2009 à 2017 (figure 4) montre qu'en dix ans, la durée de séjour portuaire est divisée par 2. Cette avancée significative est le fait des meilleurs rendements 
constatés dans la manutention avec le concours de DP World (Diop et al., 2018) et les efforts faits pour réduire la durée les procédures de dédouanement. Le port d'Abidjan, quand à lui a de meilleurs scores dans les formalités portuaires. Là où, au port de Dakar on peut attendre 6 jours, elles ne dépassent presque jamais 48 heures à Abidjan et les formalités douanières prennent une journée.

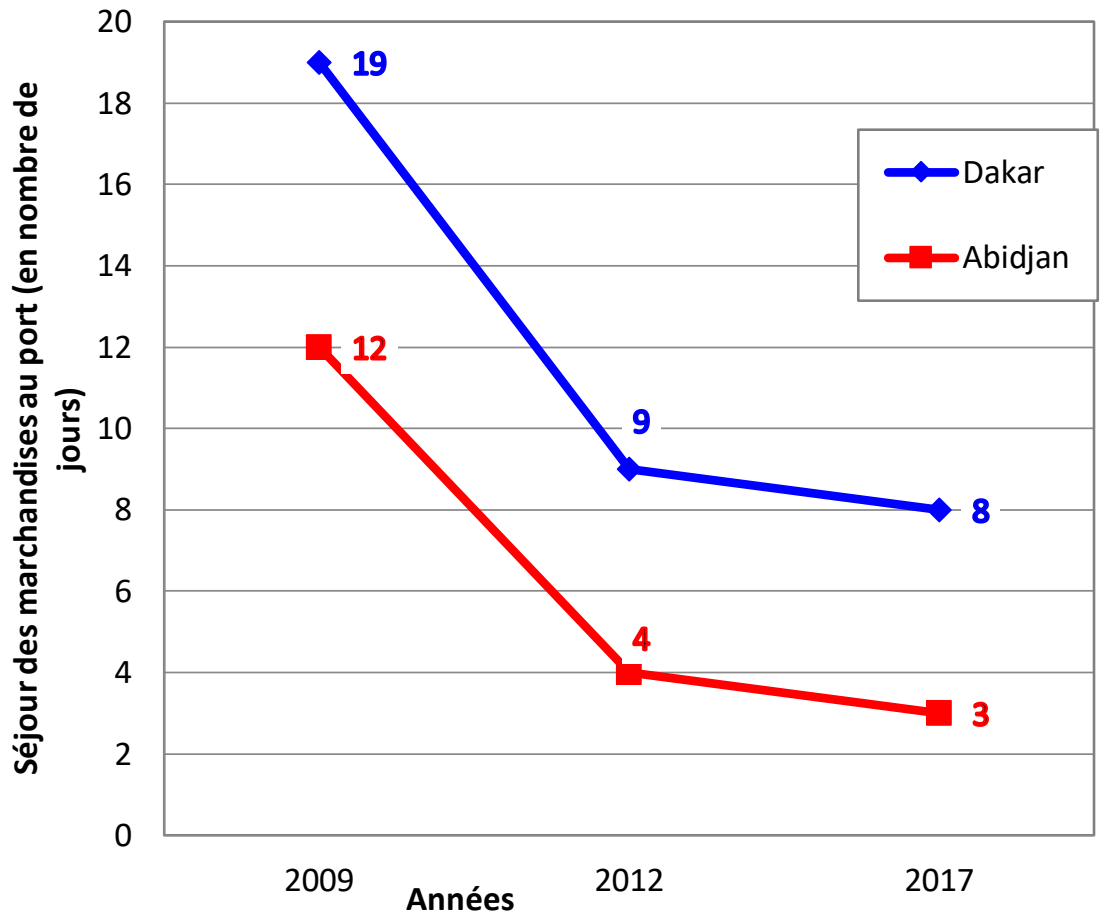

Figure 4 : Temps de passage portuaire aux ports de Dakar et d'Abidjan de 2009 à 2017.

Source : I.Dia, d'après COSEC, conseil malien des chageurs; Hartmann, 2010.

Les temps de séjour portuaires des navires et des marchandises, démontrent que le port de Dakar doit prendre au sérieux le port d'Abidjan sur le marché malien (figure 4). Beaucoup d'avantages sont offerts au Mali pour le fidéliser, certes, mais Dakar devra s'ajuster surtout en termes d'infrastructures et de délais de passage portuaire car ce sont des éléments importants sur lesquels, les chargeurs (au même titre que les armateurs) évaluent les ports (Fassio et Le Mestre, 2009)

\subsection{L'analyse des dispositifs de desserte du port de Dakar vers Bamako}

Aucun port ne prospérerait s'il ne dispose pas de desserte terrestre à son aval. L'étude des corridors de desserte du port de Dakar vers Bamako met en exergue des problèmes liés aux matériels et aux infrastructures, mais 
également aux caractéristiques non physiques, notamment, à la gouvernance du corridor, ce qui, naturellement impacte la compétitivité du port.

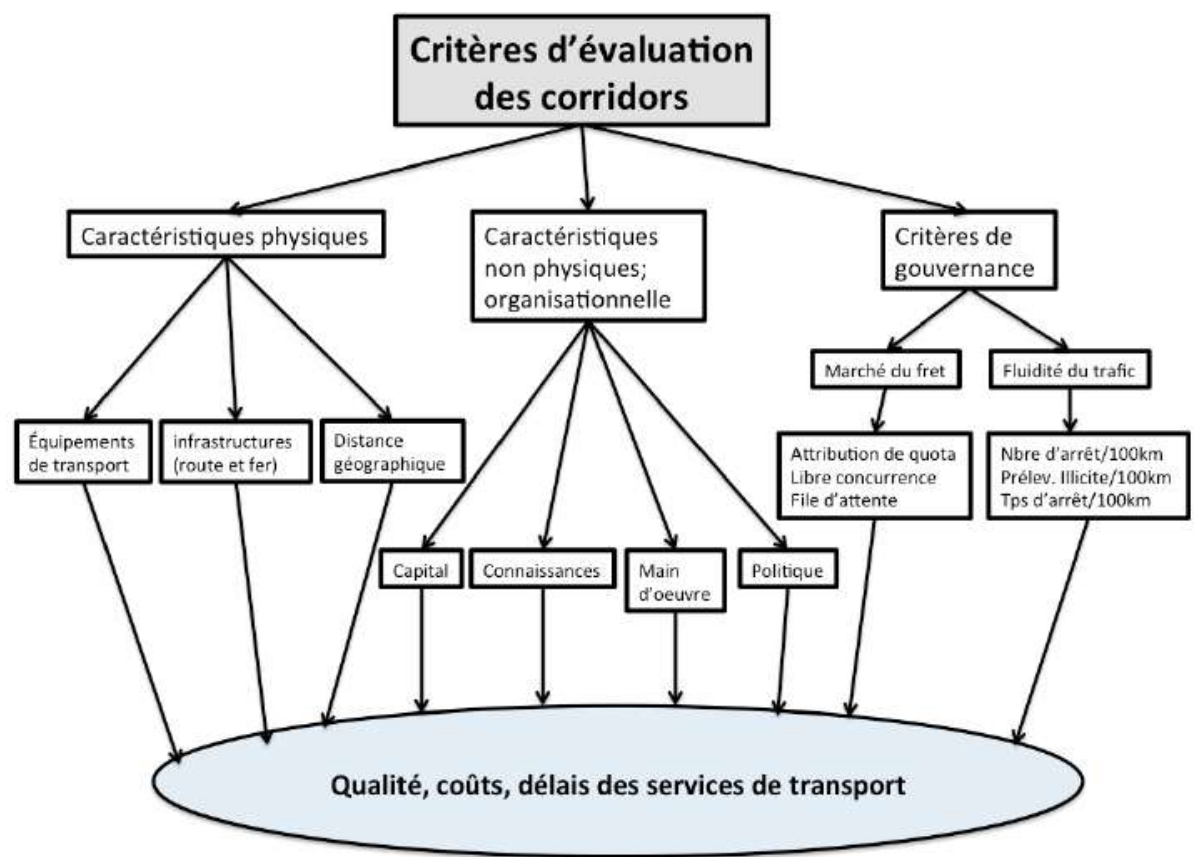

Figure 5: Critères d'évaluation des corridors

Source : I.Dia, adapté de Dicko (2018).

Tableau 5 : Caractéristiques du corridor Dakar-Bamako

\begin{tabular}{|c|c|}
\hline Linéaire en km & $\begin{array}{l}\text { - Route : } \\
\quad \text { • axe nord : } 1382 \\
\text { • axe sud : } 1225 \\
\text { - Voie ferrée (écartement métrique) : } \\
\quad \text { • } 33 \text { gares et stations de Dakar jusqu'à Koulikoro } \\
\quad \text { • } 1233 \text { dont } 644 \text { au Sénégal ; } \\
\text { Accès direct aux terminaux portuaires de DP World et aux } \\
\text { plateformes logistiques à Dakar et à Bamako (EMASE et } \\
\text { ENSEMA) }\end{array}$ \\
\hline Principaux centres urbains & $\begin{array}{l}\text { - Route : Dakar, Kaolack, Tambacounda (Sénégal) : } \\
\text { - Kidira-Diboli (frontière) - Kayes, Bamako (Mali) } \\
\text { - Kédougou-Saraya-Moussala (frontière) }- \text { Kita, } \\
\text { Bamako (Mali) } \\
\text { Chemin de fer : Dakar, Guinguinéo (embranchement } \\
\text { Kaolack-Guinguinéo), Tambacounda, Kidira (frontière) - } \\
\text { Kayes, Kita, Bamako }\end{array}$ \\
\hline Principaux produits transportés & $\begin{array}{l}\text { - Riz, coton, engrais, ciment, sel, produits pétroliers, } \\
\text { céréales, produits divers }\end{array}$ \\
\hline
\end{tabular}




\begin{tabular}{|c|c|}
\hline $\begin{array}{l}\text { Coûts de transport de marchandises } \\
\text { (ex : riz) depuis le port, toutes charges } \\
\text { comprises (douanes sénégalaises et } \\
\text { maliennes, transport, manutention, } \\
\text { traversée frontière, autres) pour un } \\
\text { conteneur de } 20 \text { pieds et un } \\
\text { chargement de } 30 \mathrm{t} \text { en vrac en USD }\end{array}$ & $\begin{array}{l}\text { - Route : } \\
\text { - conteneur } 20 \text { pieds/route : } 4150 \\
\text { - } 30 \text { tonnes en vrac/route : } 3348 \\
\text { - Rail : conteneur } 20 \text { pieds/rail : } 2703\end{array}$ \\
\hline $\begin{array}{l}\text { Caractéristiques des matériels } \\
\text { roulants }\end{array}$ & $\begin{array}{l}\text { - Environ } 400 \text { véhicules/jour ( } 85 \% \text { maliens), } 60 \% \text { des } \\
\text { transporteurs n'ont qu'un véhicule, la flotte est plus } \\
\text { récente au Mali qu'au Sénégal où } 85 \% \text { des véhicules ont } \\
\text { plus de } 10 \text { ans. } \\
\text { - Depuis } 2017 \text {, aucune locomotive n'est en circulation } \\
\text { pour un parc théorique de } 23 \text { unités dont } 12 \text { utiles. } \\
\text { - Un recensement fait en octobre } 2017 \text { donne un parc de } \\
\text { wagons théorique de } 442 \text { dont } 417 \text { en service. }\end{array}$ \\
\hline $\begin{array}{l}\text { Aires de stationnement pour gros } \\
\text { porteurs }\end{array}$ & $\begin{array}{l}\text { - } 3 \text { aires de stationnement ont été construites depuis } 2009 \\
\text { sur le corridor à Kaffrine, Koungheul et Missira. Jusqu'à } \\
\text { présent, pas encore mis en service. } \\
\text { - Parc gros porteur de Diamniadio qui a une capacité de } \\
200 \text { places sur } 9 \text { ha avec possibilité d'extension }\end{array}$ \\
\hline Acteurs/parties prenantes & $\begin{array}{l}\text { - Internes : dirigeants, syndicats, camionneurs formels \& } \\
\text { informels, transitaires, agents de douanes, etc. } \\
\text { - Externes: autorités des pays (Sénégal et Mali), } \\
\text { institutions régionales et bailleurs de fonds : } \\
\text { Communauté Economique des Etats de l'Afrique de } \\
\text { l'Ouest (CEDEAO), Banque Islamique de } \\
\text { Développement (BID), Banque Africaine de } \\
\text { Développement (BAD), Banque Ouest Africaine de } \\
\text { Développement (BOAD), Japan International } \\
\text { Cooperation Agency (JICA), Fonds saoudien de } \\
\text { développement (FSD) etc. }\end{array}$ \\
\hline
\end{tabular}

Source : I. Dia, d'après DBF, AGEROUTE, Port de Dakar, Direction des transports

Le corridor Dakar-Bamako offre des possibilités de choix d'itinéraire (route) et de mode de transport. Par la route, le corridor dessert et participe au dynamisme économique de certaines localités à l'exemple de Kaolack ou de Kayes. Il révèle également, à travers l'axe sud du corridor, certaines localités qui étaient peu connues comme l'axe Saraya-Moussala. L'effet contraire est entrain de se constater sur le corridor ferroviaire où les moments de rush coïncidaient avec les passages des trains. Aujourd'hui par exemple Guinguinéo, qui est une gare principale, s'oublie de plus en plus suite aux difficultés constatées du chemin de fer.

Le post-acheminement routier est très sollicité, c'est d'ailleurs le seul mode actuellement fonctionnel. On y dénombre plus de 400 véhicules/jour en moyenne quittant le port de Dakar (port autonome de Dakar, 2017). Lors des enquêtes de terrain en février 2018, précisément le 10, entre 18 et 19 heures (fin de journée), 79 camions ont été comptés à l'entrée de Tambacounda. Le 
même mode opératoire a été reproduit cette fois à l'entrée de Kidira (axe nord) le 14 février entre 13 et 14 heures et entre 18 et 19 heures, les résultats trouvés sont respectivement de 19 et de 37 camions. Cette même manœuvre a été reconduite une troisième fois à Moussala le 21 février. Aux observations de 14 heures 17 camions étaient passés et à 19 heures, 32 .

Ces résultats montrent combien est sollicité le corridor routier et combien il est urgent de trouver des solutions aux problèmes d'infrastructures, de gouvernance et de gestion tant du côté routier que du côté ferroviaire (qui est en phase de dégénérescence avancée) afin de répondre de façon efficace à l'appel de l'hinterland malien.

\subsubsection{La situation actuelle de la desserte routière}

\subsubsection{Les critères matériels et infrastructurels sur le corridor}

L'état des infrastructures routières sur le corridor Dakar-Bamako est satisfaisant à part des dégradations constatées sur le tronçon TambacoundaKidira, pour lesquelles des travaux de réhabilitation sont engagés. La première phase de ces travaux concernant l'axe Tambacounda-Goudiry $(80 \mathrm{~km})$ a été lancée en août 2018. Ils sont financés par le FSD et le gouvernement du Sénégal. Du côté malien, l'axe Diboli-Kayes-Diéma-Kati est également en mauvais état. Le gouvernement malien a lancé un projet de réhabilitation de ce tronçon depuis octobre 2018 dont la première phase de celui-ci couvre l'axe Didiéni à Kati (qui est à l'entrée de Bamako). Sur l'axe sud du corridor, le tronçon Tambacounda-Dialocoto, financé par l'Etat du Sénégal et la BOAD, a été inauguré en 2018. Elle est suivie par la réhabilitation de l'axe DialacotoMako, qui est en cours, et dont le financement est assuré par la BID, l'État du Sénégal et la BOAD.

Disposer de bonnes infrastructures routières est la base d'une bonne desserte routière. Elles nécessitent cependant une gestion et un entretien rigoureux de ces ouvrages. Les matériels (gros porteurs) surchargés qui détruisent les routes en quelques mois sont l'un des principaux points faibles dont il fallait trouver des solutions (Bove et al., 2018). Depuis le $1^{\text {er }}$ septembre 2016, le règlement $\mathrm{N}^{\circ} 142005 \mathrm{du}$ Conseil des ministres de l'UEMOA a pris effet. Son rôle est de lutter contre l'extrême surcharge et le dépassement des gabarits autorisés sur les routes. Pour ce faire, des postes de pesage ont été installés sur les routes pour l'application effective de cette mesure.

Une autre faiblesse de la desserte routière est le parc de gros porteurs qui est au désavantage du Sénégal avec $85 \%$ de la flotte qui est malienne (tableau 5). Le Sénégal souffre également d'un parc dont $85 \%$ des véhicules ont au moins 10 ans (Bove et al., 2018). Dans une optique de rajeunir ce parc, le gouvernement sénégalais a interdit l'importation de véhicules de plus de dix ans (huit ans pour les véhicules légers) et mène un dialogue avec les opérateurs économiques par l'intermédiaire de la coopérative nationale des entreprises de 
transport de marchandises (CNETM), afin de proposer des options de financement pour le renouvellement du parc automobile. Le but fixé est un renouvellement du parc camions sénégalais, avec un objectif assigné de 3000 unités entre 2016 et 2022 pour un montant de 400 millions de dollars environ. Une phase pilote de 1600 véhicules a été lancée en 2016 et financée par la Banque Nationale pour le Développement Économique (BNDE) accompagnée d'une garantie de l'État. Dans ce cadre 800 camions avaient été remis à la CNETM en février 2016. En décembre 2018, 73 autres camions avaient été livrés. Un troisième lot de 150 camions était en préparation. L'octroi de ces véhicules aux transporteurs est basé sur leur enregistrement dans les bureaux régionaux de la CNETM. L'allocation des fonds pour les véhicules est effectuée en fonction de l'ordre d'inscription, ce qui ne donne pas malheureusement la priorité aux véhicules les plus âgés, donc diffèrera leur sortie du circuit. La lutte contre la vétusté des véhicules (Villamil Galvis, 2014) et une exploitation responsable des flottes (lutte contre les surcharges et la maintenance dans les délais) peuvent, certes, réduire les charges d'exploitation des camions, donc des prix de vente de la prestation de transport, mais surtout être des actes préventifs d'entretien qui font partie des parents pauvres de la gestion des infrastructures africaines.

Sur le corridor dakar-Bamako, les aires de stationnement font défaut. Le précédent gouvernement du Sénégal (2000-2012) avait construit 3 parkings en 2009 respectivement à Kaffrine, Koungheul et Missira (sur 1'axe KaolackTambacounda). Dix ans après, ils sont toujours fermés malgré les promesses faites sur leur mise en service. Dans ce contexte, une petite enquête a été menée sur le sujet, mais les autorités politiques semblent éviter la question. Quoiqu'il en soit, leurs ouvertures auraient permis de réduire les accidents de la circulation causés par les stationnements des gros porteurs le long de la route qui, sont aussi responsables de la dégradation des infrastructures. Elles auraient également permis d'empêcher des stationnements sauvages et sans autorisation sur des terrains vagues comme celui qui est à l'entrée de Sandiara (ville entre Mbour et Fatick). Heureusement qu'en début d'année 2019, dans le cadre des projets du Plan Sénégal Emergent (PSE), la gare des gros porteurs de Diamniadio a été inaugurée (tableau 5). Cette gare est à la croisée des chemins et s'ouvre sur les principaux itinéraires routiers à l'intérieur du pays, à l'exemple du corridor Dakar-Bamako. 


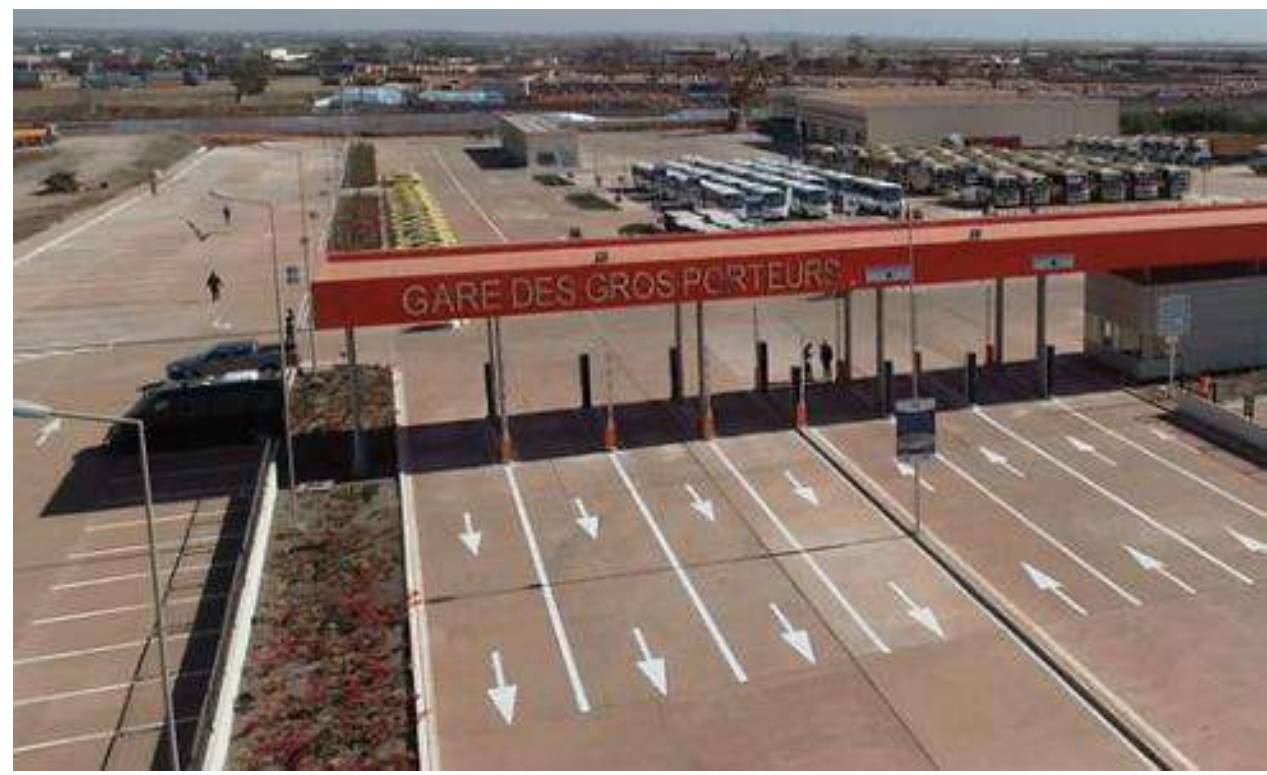

Photo : Gare des gros porteurs de Diamniadio (à $40 \mathrm{~km}$ de Dakar). Source : www.flickr.com

\subsubsection{La gouvernance du corridor routier}

Les textes qui régissent les transports routiers en Afrique de l'Ouest adoptés par les États membres de la CEDEAO ont été repris dans le cadre de l'UEMOA. Ils définissent un cadre harmonisé concernant les normes de circulation. Sur le corridor Dakar-Bamako, on note la non-application correcte des conventions sur le transport inter-Etats et de transit (CEDEAO et le TRIE - Transit Routier Inter Etats -). Cela crée des situations de nonefficacité des trafics comme :

- de mauvaises interprétations et applications des outils institutionnels et administratifs ;

- des contrôles administratifs (scanner, ponts bascules) et des demandes de documents et des vérifications répétées (en cours de route et à la frontière), motivées par le seul souci d'effectuer des prélèvements souvent illicites sans oublier l'impact négatif que cela a sur la durée du transit routier (BAD, 2015). 
Tableau 6: état des postes de contrôles par corps et par voyage sur le corridor

Dakar-Bamako

\begin{tabular}{|c|c|c|c|c|c|c|c|c|c|c|}
\hline \multirow{2}{*}{$\begin{array}{l}\text { Corridor } \\
\text { Dakar- } \\
\text { Bamako }\end{array}$} & \multirow{2}{*}{$\begin{array}{l}\text { Dist } \\
\text {. en } \\
\text { km }\end{array}$} & \multicolumn{8}{|c|}{ Nombre moyen de poste de contrôle/corps/voyage } & \multirow[b]{2}{*}{$\begin{array}{l}\mathbf{N} \\
\mathbf{C}\end{array}$} \\
\hline & & $\mathrm{P}$ & $\mathrm{D}$ & $\mathrm{G}$ & $\mathrm{EF}$ & $\mathrm{Ph}$ & Autres & Total & $\begin{array}{l}\text { Ratio sur } \\
100 \mathrm{~km}\end{array}$ & \\
\hline Via Kidira & $\begin{array}{l}138 \\
2\end{array}$ & 7 & 5 & 10 & 0 & 1 & 3 & 26 & 2 & 3 \\
\hline Via Moussala & $\begin{array}{l}122 \\
5\end{array}$ & 6 & 5 & 6 & 1 & 2 & 0 & 20 & 2 & 3 \\
\hline
\end{tabular}

*P : Police ; D : Douane ; G : Gendarmerie ; EF : eaux et forêts ; Ph :Phytosanitaire ; Autres : mairies, syndicats, impôts ; NC : norme communautaire.

Source: Rapport OPA (Observatoire des pratiques anormales)UEMOA de 2017 et confrontation par un décompte sur le terrain (enquêtes du 02 au 28 fevrier 2018).

Tableau 7: perceptions illicites par véhicule par voyage et par corps de contrôle sur le corridor Dakar-Bamako (en FCFA).

\begin{tabular}{|l|lllll|}
\hline $\begin{array}{l}\text { Corridor } \\
\text { Dakar- } \\
\text { Bamako }\end{array}$ & \multicolumn{5}{|l|}{ Réception par corps/ voyage/véhicule } \\
\cline { 2 - 6 }
\end{tabular}

\begin{tabular}{l|l|l|l|l|l|}
\hline Via Kidira & 12350 & 9900 & 20100 & 2000 & $\mathbf{4 4 3 5 0}$ \\
\hline Via Moussala & 16400 & 10320 & 15575 & 3000 & $\mathbf{4 5 2 9 5}$ \\
\end{tabular}

Tableau 8: temps moyen de contrôle en minutes / véhicule/ voyage ; $($ Ratio = temps de contrôle/ distance corridor/ $100 \mathrm{~km}$ )

\begin{tabular}{|l|l|l|}
\hline \multirow{2}{*}{ Corridor Dakar-Bamako } & Contrôle en minutes \\
\cline { 2 - 3 } & $\begin{array}{l}\text { Temps de contrôle sur } \\
\text { tout le corridor }\end{array}$ & $\begin{array}{l}\text { Ratio en mn de contrôle/100 } \\
\mathrm{km}\end{array}$ \\
\hline Via Kidira & 299 & 21,6 \\
\hline Via Moussala & 251 & 20,4 \\
\hline
\end{tabular}

Source : I. Dia, adapté du rapport OPA, 2017.

Suite aux mauvais résultats du rapport de l'OPA de l'UEMOA (6 fois le nombre de contrôle conseillé, des perceptions illicites des agents de contrôle ainsi que des temps de contrôle longs), le Sénégal s'est engagé à réduire et à harmoniser le nombre de points de contrôle entre Dakar et Kidira (frontière avec le Mali). La gendarmerie a adopté diverses mesures anti-corruption, telles que la mise en service d'un numéro vert pour signaler les points de contrôle illégaux, le port de badges d'identification pour les agents et des dispositifs GPS pour une meilleure localisation des agents sur le terrain, etc. Cette initiative vise également à réduire au nombre de trois (recommandation de l'UEMOA) les points de contrôle (y compris les ponts bascules pour l'application des règlementations UEMOA relatives à la charge à l'essieu). 


\subsubsection{Un chemin de fer complètement sous l'ombre du transport routier}

DBF est une société binationale disposant d'une voie unique de Dakar à Bamako. Depuis l'avènement du train express régional (TER), les emprises de la deuxième voie lui sont affectées rendant la voie double Dakar-Thiès une voie unique. Les infrastructures fixes et le matériel roulant sont dans un état de vétusté avancé, ce qui causait de nombreux déraillements (environ 100 en 2016 selon DBF, 2016) et expliquait la vitesse moyenne de $20 \mathrm{~km} / \mathrm{h}$ qu'on y constatait. A certains tronçons où il $\mathrm{y}$ avait des ponts dégradés ou zones ensablées, la vitesse pouvait descendre parfois en deçà de $10 \mathrm{~km} / \mathrm{h}$. La durée moyenne d'une expédition depuis Dakar était d'environ 72 heures (statistiques de DBF, 2017). Ces situations désastreuses sur la voie, doublées d'un parc locomotives désuet (23 en 2003 et 0 en 2017 ; DBF, 2019), ont conduit à l'arrêt du trafic Dakar-Bamako.

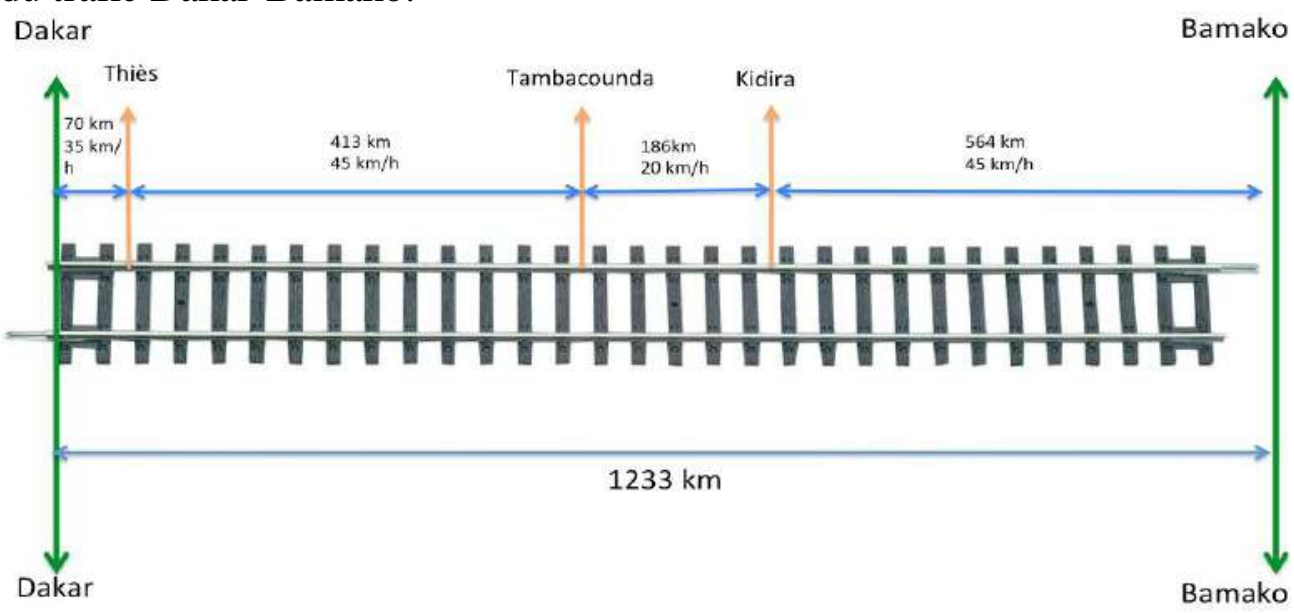

Figure 6 : Estimation moyenne des plus grandes vitesse atteintes sur le corridor ferroviaire

Dakar-Bamako par tronçon de 2012 à 2017.

Source : I. Dia, d'après les enquêtes de terrain

Les figures 6 et 7 synthétisent les pointes de vitesse sur le corridor de 2012 à 2017 et la chute libre du trafic. 


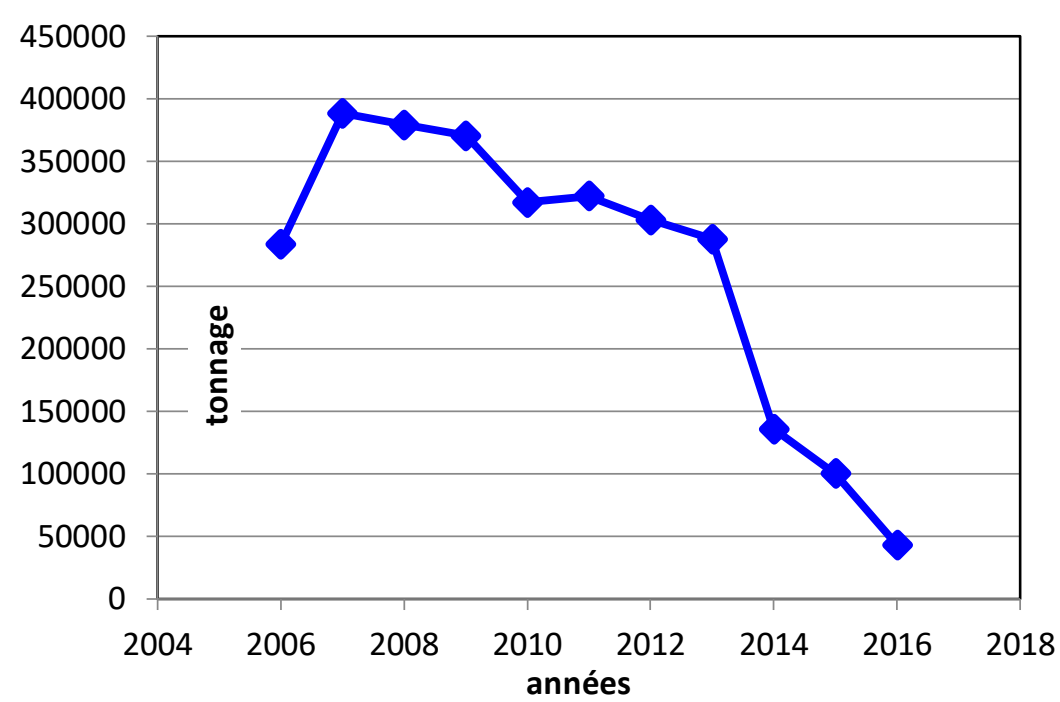

Figure 7 : Trafic annuel de 2004 à 2016 (Transrail et DBF) Source : I. Dia, d'après les statistiques collectées à DBF

Le chemin de fer, pourtant, revêt une importance capitale pour le Mali et le Sénégal. Il est même urgent de le rendre à nouveau opérationnel car c'est un maillon important de chaîne logistique entre les deux pays :

- Pour le Sénégal : un DBF fonctionnel permettra au port d'accroître son trafic, de mettre fin à la congestion (qui fait partie des priorités de l'actuel directeur du port de Dakar), d'accroître la longévité de la route, de rendre le port plus compétitif ; etc.

- Pour le Mali, le corridor pourra jouer un rôle important de désenclavement et de rabais des coûts de post-acheminement complètement sous l'apanage de la route (contrôle de la police, douane et gendarmerie, qui se matérialise par une perte de temps et des dépenses supplémentaires, le coût du transport etc.)

Le corridor ferroviaire Dakar-Bamako a un potentiel inestimable certes, mais inemployé car nécessitant des investissements considérables pour réellement changer la desserte du port de Dakar. Le Mali est convoité par quasiment tous les ports de la rangée Nouadhibou (Mauritanie) à Cotonou (Bénin). Le port de Dakar, en terme de flux, dépasse de loin ses concurrents avec un trafic en transit malien qui fait 3,25 fois celui de son challenger direct ; le port autonome d'Abidjan (PAA). Ne serait-il pas opportun de pour le Sénégal de trouver des solutions aux problèmes qui gangrènent le fonctionnement de DBF afin de consolider son avance ? Les autorités politiques sont conscientes du manque à gagner pour le port (chiffre d'affaires, compétitivité etc.), c'est d'ailleurs dans ce sens qu'un nouveau directeur a été nommé à la tête de DBF. Le nouvel administrateur lors d'une interview 
accordée à la Radio France Internationale (RFI) en décembre 2018, emboitait le pas de sa hiérarchie en rappelant que : «(...) la liaison Dakar-Bamako va refonctionner rapidement (...) il faut redonner à l'entreprise sa dignité en faisant que les trains aillent et viennent ».

Le train, s'il était fonctionnel, pourrait avec la route fournir des services de transport complémentaires ou créer une concurrence positive à l'avantage des usagers.

Il faut toutefois reconnaître que le chemin de fer est dans un niveau de dégradation très avancée dont la remise en service nécessite une grande volonté. La nouvelle équipe dirigeante de DBF parle d'un ticket d'entrée de 200 millions de dollars ou davantage, si elle prend en compte la rénovation des gares, pour un retour optimal du chemin de fer. Mais dans l'urgence, le nouveau directeur atteste de l'acquisition de 11 locomotives, assez pour que dans un court délai, les activités reprennent.

\section{Conclusion}

Les ports de Dakar et d'Abidjan sont les portes océanes les plus sollicitées pour le commerce extérieur malien. Le choix de ces ports n'est pas dû au hasard. Il dépend des performances des ports, des tarifs qui y sont appliqué mais aussi aux corridors (l'état des routes). Si les systèmes de transport en Afrique de l'Ouest ne répondent pas encore aux standards mondiaux, des efforts sont notés surtout sur les infrastructures routières. Cela se matérialise sur le corridor Dakar-Bamako qui présente des atouts susceptibles d'accroître son rôle dans la compétitivité du port de Dakar. Le corridor Dakar-Bamako dispose également d'un transport ferroviaire qui rencontre des difficultés depuis quelques années, en témoigne la chute libre de son trafic (figure 7) jusqu'en 2017 où on a constaté un arrêt des activités transport à cause d'un défaut de locomotive fonctionnelle. Malgré cette moins value causée par le chemin de fer, le port Dakar devance encore ses concurrents sur le marché malien. Mais ce qu'il ne faudrait pas négliger cependant, ce sont les investissements en cours au port d'Abidjan (principal concurrent du port de Dakar sur le marché malien) et les rencontres entre les autorités ivoiriennes, celles du port d'Abidjan, de San Pedro et des opérateurs économiques maliens (conseil malien des chargeurs et le conseil malien des transporteurs routiers) qui peuvent à terme être préjudiciables à Dakar. Le défi du port de Dakar, c'est de rester à la place du leader, et pour cela, un sérieux diagnostic de sa compétitivité est important. La compétition portuaire se passe sur l'hinterland. Beaucoup d'efforts sont faits pour le transport routier tant sur le plan infrastructurel (construction de routes, réhabilitation/rénovation), que matériel (renouvellement du parc des gros porteurs, en cours). Des efforts sont également faits sur la gestion du transport sur le terrain (avec des mesures prises pour lutter contre les pratiques anormales. Ce sera un combat de longue 
haleine). Par ailleurs, il est impossible de traiter la question de la desserte du port de Dakar sans s'indigner sur la situation actuelle du chemin de fer, qui est un vrai manque à gagner pour les deux pays. Depuis quelques années, sa mise en service est annoncée par les hautes autorités des deux pays. Jusqu'à présent, elle reste dans le cadre des promesses non encore tenues.

Le port de Dakar mise sur sa desserte pour être plus compétitif. Qu'en sera t-il des mesures prise pour l'amélioration de la desserte routière ? Le chemin de fer est dans une phase de restructuration institutionnelle et fonctionnelle. Il faudra donc lui laisser le temps de renaître et de redevenir opérationnel pour pouvoir contribuer de nouveau à la compétitivité du port de Dakar.

\section{References:}

1. Aronietis, R., Markianidou, P., Meersman, H., Pauwels, T., Pirenne, M., Van De Voorde, E., Vanelslander, T. \& Verhetsel, A. (2010). Some effects of hinterland infrastructure pricing on port competitiveness: case of Antwerp. 12th WCTR, Lisbon, Portugal. 23p.

2. BAD. (2015). Problématique de la facilitation du transport routier en Afrique de l'ouest et plans d'actions. Département des transports, du développement urbain et des TICS; 54p.

3. Bove, A., Hartmann, O., Stokenberga, A., Vesin, V. \& Yedan, Y., (2018). Le transport routier en Afrique de l'Ouest et en Afrique Centrale. SSATP, Programme des politiques de transport en Afrique. Document de travail $\mathrm{N}^{\circ} 108.153 \mathrm{p}$.

4. CEA. (2004). Accélérer l'intégration régionale en Afrique: vue d'ensemble. United Nations Publications, 36 p.

5. Champonniere, J-R. (2008). Un démi-siècle de relations ChineAfrique : évolution des analyses. In Afrique contemporaine, 2008/4, 228, pp 35-48.

6. Dicko, A. H. (2018). Compétitivité portuaire et desserte terrestre : les corridors de désenclavement du Mali. In Moderniser les ports ouestafricains : enjeux et perspectives, EMS, pp 295-317.

7. Diop, N., Pouye, N.N. \& Mbengue, R. (2018). Partenariat public-

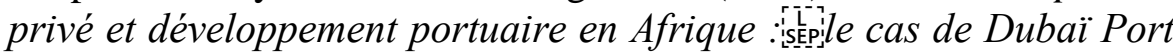
World au port de Dakar. In Moderniser les ports ouest-africains : enjeux et perspectives, EMS, pp 173-185.

8. Flavient, D. L., (2018). La redynamisation des ports ivoiriens. In Revue Espace géographique et société marocaine, $\mathrm{n}^{\circ} 24-25.16$ p.

9. Fassio, G. Le mestre, P. (2009). Réalités organisationnelles des places portuaires en France et absence d'une mesure tri-dimensionnelle (Coûts - Qualité - Délais) de leur performance. Journées d'étude en contrôle de gestion de Nantes, journée : - La gestion conjointe des 
coûts, de la qualité et des délais 30 janvier 2009 - IEMN-IAE Université de Nantes. 15p.

10. Harding, A., Raballand, G. \& Pálsson, G. (2007). Ports et transport maritime en Afrique de l'ouest et du Centre : les défis à relever. Document de travail SSATP n ${ }^{\circ} 84 \mathrm{~F}$, The World Bank, 56 p.

11. Krugman, P. (1998). La mondialisation n'est pas coupable. Vertus et limites du libre-échange. Editions La Découverte, Paris, 218 pages.

12. Lihoussou, M. (2014). Port et désenclavement territorrial : cas de l'arrière pays du port de Cotonou. Thèse de doctorat de Géographie, Spécialité : Transport et Logistique. Université du Havre, Ecole doctorale 556 Homme, Sociétés, Risques Territoires. UMR IDEES, 6266 CNRS/CIRTAI.

13. Maloni, M., Jackson, E.C. (2005). North American container port capacity : a liiterature review. In the Transportation journal.

14. Matz, E. (2017). Edito: Le secteur portuaire en Afrique. SP\&D, N²6, Proparco, Groupe AFD, p3.

15. Merk, O., Comtois, C. (2012). Compétitivité Des Villes Portuaires: Le Cas De Marseille-Fos. OECD Regional Development Working Papers, 2012/11, OECD Publishing, Doi : 10.1787/5k8x9b79bmlw-fr

16. Meyer, G. (2017). L'insertion des ports africains dans les flux mondiaux : atouts et faiblesses. SP\&D, N²6, Proparco, Groupe AFD, pp22-25.

17. OECD/ITF. (2008). Port competition and hinterland connections : summary and conclusions. Working paper ; Transport Research Centre Discussion Paper, $N^{\circ}$ 2008-19.

18. Onwuegbuchunam, D.E. (2013). Port selection criteria by shippers in Nigeria: a discrete choice analysis. Int. J. Shipping and Transport Logistics, Vol. 5, Nos. 4/5, 2013.

19. Port autonome d'Abidjan - PAA -. (2008). Barème des redevances portuaires. PAA, 35p.

20. Port autonome de Dakar - PAD -. (2006). Barème des redevances portuaires. PAD, 44p.

21. PAD. (2014). Note circulaire à l'attention des usagers du port de Dakar: institution d'une redevance sureté ISPS. PAD, Direction générale, 3pRFI (Radio France Internationale). (2018). Emission radio, http://www.rfi.fr/video/20181203-quai-orsay-dakar-bamakoferroviaire-kibily-toure-grand-invite-economie-1

22. Solagral. (2003). Etude sur des mesures de reduction de la volatilite des prix des matieres premieres. hubrural.org/IMG/pdf.

23. Steck, B. (2004). La mondialisation et risque de la fragmentation territoriale. le cas d'un Etat enclavé du sud: le Mali (Afrique de l'Ouest). Belgéo, $17 \mathrm{p}$ 
24. UEMOA. (2017). 28 $8^{\text {ème }}$ rapport de l'observatoire des pratiques anormales (OPA) : $2^{\text {ème }}$ trimestre de l'année 2017. UEMOA, 13p.

25. UNCTAD. (2018). Review of maritime transport. United Nations, New York and Geneva, $102 \mathrm{p}$.

26. URD. (2008). Operating regulations and tariff schedule for the port of Dakar. URD and Boluda, France. 4p.

27. Villamil Galvis, G. E. (2014). Outils D'aide A La Décision Pour Le Renouvellement D'une Flotte De Véhicule. Mémoire de maîtrise en Génie mécanique, Université LAVAL, Québec, Canada.

28. Yao Beli, D., Konan Koffi, C. (2018). Le transport de marchandises entre le port d'Abidjan et les pays de l'hinterland. In Moderniser les ports ouest-africains : enjeux et perspectives, EMS, pp 319-335.

29. Zhang, A. (2009). Impact des conditions d'accès à l'hinterland sur la rivalité interportuaire. In Concurrence entre les ports et les liaisons terrestres avec l'arrière-pays., Table ronde FIT, OECD. Paris ; pp 142175.

30. http://www.portdakar.sn/fr/nous-decouvrir/nos-chiffres-cles

31. http://www.portabidjan.ci/fr/le-port-dabidjan/statistiques-portuaires 